\title{
Introduction to COX inhibitors
}

\author{
Mariangela Biava*,1 \\ ${ }^{1}$ Dipartimento di Chimica e Tecnologie del Farmaco, Sapienza University, P.le A. Moro, 5 Roma 00185, Italy \\ *Author for correspondence: mariangela.biava@uniroma1.it
}

First draft submitted: 4 May 2018; Accepted for publication: 23 May 2018; Published online: 18 July 2018

It is well known that COX-1 and COX-2 isozymes play a central role in the biosynthetic pathway of prostanoids and exert different biological functions, determined by the differences in regulation of related gene expression, stability of transcripts and proteins. COX-1 has been considered a 'housekeeping' gene, is expressed in many tissues and cells and is responsible for physiological functions such as gastrointestinal (GI) cytoprotection, platelet aggregation and vascular smooth muscle tone modulation [1,2]. Instead, $C O X-2$ gene is upregulated during inflammation in response to IL-1 $\beta$ and other inflammatory stimuli such as mycobacterial pulmonary inflammation [3], vascular inflammation [4], cigarette-induced airway inflammation [5], Helicobacter pylori-induced gastritis, hypoxia and in many cancers [6]. However, in-depth knowledge of COX-isoforms biology has shown that also COX-1 can be upregulated in some circumstances (such as cell differentiation), while COX-2 can be constitutively expressed in physiologic conditions, such as in central nervous system, vasculature and kidney [7].

COX inhibitors can be classified into two principal classes: traditional nonsteroidal anti-inflammatory drugs ( $t$ NSAIDs) and selective COX-2 inhibitors (COXIBs). What is the link between $t$ NSAIDs and COXIBs? $t$ NSAIDs inhibit both COX isoforms, albeit with different relative potencies depending on their chemical structures and the COX-1 inhibition causes gastrointestinal adverse effects due to the several protective actions played by COX-1 in the gastrointestinal environment; as a consequence, the 'COX-2 hypothesis' claimed that at comparable COX2 inhibiting doses, COXIBs could be as effective as $t$ NSAIDs but cause less gastrointestinal adverse effects [7]. More precisely, the proof of concept was that sparing COX-1 in the GI tract, and possibly in platelets, translates into a safer profile [8]. The development of the COXIB family then represented a stimulating approach for the treatment of inflammatory disorders, such as arthritis, and for the management of acute pains, as an alternative to $t$ NSAIDs, to circumvent the side effects associated with COX-1 inhibition. Among the several synthesized COXIBs, celecoxib, SC588, rofecoxib, valdecoxib, parecoxib and later etoricoxib and lumiracoxib were the most active ones. Unfortunately, rofecoxib and valdecoxib, the most selective ones, were withdrawn from the market over an increased incidence of thrombotic events associated with their long-term use [9]. Unexpectedly, it was observed that a high selectivity toward COX-2 was related to a high risk of cardiovascular $(\mathrm{CV})$ undesirable effects. This observation is supported by the fact that celecoxib, which has the lowest COX-2/COX-1 selectivity index [10] is the only COXIB still approved by the US FDA. Moreover, on the other hand the incidence of thrombotic events can be mitigated, even if not abolished, by a complete suppression of platelet COX-1 activity [11].

\section{Finding the perfect balance}

Is there an anti-inflammatory drug able to completely suppress the platelet COX-1 activity and at the same time be nontoxic for the GI system? Most $t$ NSAIDs and COXIBs are not able to completely and persistently inhibit platelet COX-1 activity and consequently to determine an in vivo inhibition of platelet function [11,12]; if that happens, as in the case of naproxen, the inhibitor on the one hand shows a profound platelet COX-1 activity inhibition and on the other a high toxicity for the GI system due to its ability to inhibit the production of cytoprotective GI prostanoids. It seemed a road without an end.

However, a marked intersubject variability in the response both to tNSAIDs and COXIBs highlighted the need of new therapeutic agents to provide a valuable treatment option for each individual patient, and many novel scaffolds have been then identified to obtain inhibitors with a balanced activity toward the two isozymes.

From a chemical point of view, tNSAIDs can be grouped into three main classes: carboxylic acids; phenazone and nonacidic compounds [13]. 
While considerable efforts have been made to identify new selective COX-2 inhibitors, very few have been done to identify COX-1 selective ones, probably due to the scarce knowledge of COX-1 biology and its involvement in human diseases. Recently, Scilimati et al. reported that GI adverse effects are mistakenly associated with selective COX-1 inhibition: indeed they demonstrated that some of 3-(5-chlorofuran-2-yl)-5-methyl-4-phenylisoxazole derivatives, highly selective toward COX-1, were devoid of GI toxicity [13]. From a chemical point of view, they affirmed that both the presence of the carboxylic group in one of them, the mofezolac, and the presence of a halogen in one were relevant. Such substituents are very important for the activity, and are essential to make contacts with the active site channel, while the bulkiness and chemical features of substituents linked to the central heterocycle, the isoxazole, appear to control the affinity for the COX-1 channel.

Selective COX-2 inhibitors, in general, are structurally unrelated to $t$ NSAIDs. Indeed, COXIBs are endowed with a bulkier aromatic ring that allows them to interact only with the bigger COX-2 hydrophobic channel [13]. They are mainly characterized by a diaryl etherocyclic scaffold, and the largest proportion of them comprises a five-membered core and diarylheterocycles, decorated with either a sulfonamide or a methylsulfamoyl group as well as with small electron-withdrawing substituents. The five-membered cores are generally represented by pyrazole, dihydropyrazole, pyrazoline, 1,2,3-triazoles, pyrrole, 2-imidazoline, tetrazole, oxadiazole and thiadiazole [14]. They can also be replaced by indols, in which the two aryl rings are connected to a tricyclic core in the forms of imidazopyrazolopyridine or dihydropyrrolo- or pyrrole-[3,2,1-hi] indole (the most active one probably because a more extended $\pi$-system electronic influences activity). Methylsulfamoyl and sulphonamide are always present in these structures, as well as small electron-withdrawing groups, that contribute to enhance their potencies [14].

Other derivatives were obtained by replacing the central tricyclic bridge with its bioisostere, naphthalene and chromanonespiro bridge [14]. Another new scaffold is a cyclic imide core, such as the maleimide one, combined with a benzenesulfonamide moiety (COX-2 pharmacophore). The most active derivatives of this series are characterized by a phenyl substituent at position four of the maleimide ring. Finally, new scaffolds are inspired by the nature: among them oleuropein, a phenolic bitter compound present in green olives, small peptides, flavonoids, chromen-4-one and coumarin are the most investigated ones [14].

\section{Multi-target scaffolds}

An important strategy to mitigate the CV risk associated with the COX-2 selective inhibition is to combine COXIBs with a cardioprotective component as well as to develop multi-target drugs such as: hybrid compounds, obtained by binding selective COX-2 inhibitors with moieties able to release nitric oxide (NO-COXIBs), in which the NO-COXIB portion was able to inhibit COX-2 isozyme, while vasorelaxing properties were dependent on NO release [15]; dual COX-2 and 5-LOX inhibitors in which the safer profile was due to the ability of such compounds to inhibit the production of prostaglandins from cyclooxygenase pathways and leukotrienes from lipoxygenase pathway [16]; COX-2 selective inhibitors and TP receptor antagonists. These compounds proved to inhibit both COX-1 and COX-2 isozymes and antagonize TP receptor, that is responsible of platelet aggregation, constriction of vascular smooth-muscle cells, as well as mitogenesis and hypertrophy of vascular smooth-muscle cells [17].

\section{Considering tumorigenesis}

Finally, it is important to mention that new COX scaffolds were also developed taking into account the involvement of COX isozymes in the tumorigenesis. Many COX-1 inhibitors were synthesized, starting from the knowledge that Indomethacin and its analog Sulindac have proven able to weakly inhibit cell growth, and were active in various cancer cell lines via COX independent mechanisms. That was a very useful starting point to develop new antiproliferative agents and both the carboxylic acid moiety and the benzoyl or benzylidene rings, were systematically modified. Surprisingly, even though Indomethacin did not show any tubulin inhibitory activity, the most active analog was found to inhibit a tubulin rather than COX, probably due to the lack of the carboxylic function, very important for the interaction with COXs $[13,18]$. In any case the structural features which are responsible for anti-cancer effects have not been confirmed yet.

The efficacy of many COXIBs as anti-tumor agents is currently under investigation in many clinical trials against diverse solid tumors, such as lung, ovarian, colorectal, breast, prostate and biliary-pancreatic [19].

The contribution of COX-2 to tumorigenesis is supported by both the knowledge that COX-2 overexpression is found in many very aggressive solid tumors in lung, head, neck, colorectal, breast and prostate, and by the proven efficacy of COXIBs in reducing the risk of colorectal adenoma recurrence. As COX-2 has a direct role in modulating tumor progression, celecoxib and rofecoxib were employed in different clinical trials showing efficacy 
in colonorectal tumor. However, due to the high toxicity of rofecoxib, only celecoxib is still under investigation not only in colonorectal tumor, but also in many other diverse solid tumors, such as lung, ovarian, breast, prostate and biliary-pancreatic [20].

\section{Conclusion}

Even though the COX isozymes play a pivotal role both in the inflammation process and tumorigenesis, the adverse effects associated with the long-term use of $t$ NSAIDs and COXIBs limit their prescription. There is then an urgent need for COX inhibitors with an improved therapeutic efficacy and reduced side effects. Despite the great efforts in the field, finding novel COX-2 inhibitor scaffolds with an improved therapeutic window and the right balance between COX-1 and COX-2 inhibition is challenging: many different strategies have been then developed to circumvent the CV risk associated with the COX-2 selective inhibition by combining COXIBs with a cardioprotective component and developing multi-target drugs. In spite of the pivotal role of COX-1 in the inflammatory process both in neurodegenerative disorders and in cancer (in particular in the ovarian one), very few efforts have been made to identify novel COX-1selective agents, probably due to the scarce knowledge of COX-1 biology, and more should be done to disclose the real therapeutic potential of this class of drugs.

\section{Future perspective}

Looking at the future, a valuable option might be to develop, in next 5 years, not only safer and more effective COX-inhibitor scaffolds, but also new strategies to find the 'ideal' COX therapy; the goal should be to provide the right therapeutic option by tailoring the treatment to each patient, taking into account both the medical history and the variability in the individual response to $t$ NSAIDs and COXIBs.

\section{Financial \& competing interests disclosure}

The author has no relevant affiliations or financial involvement with any organization or entity with a financial interest in or financial conflict with the subject matter or materials discussed in the manuscript. This includes employment, consultancies, honoraria, stock ownership or options, expert testimony, grants or patents received or pending, or royalties.

No writing assistance was utilized in the production of this manuscript.

\section{References}

1. Dannenberg AJ, Altorki NK, Boyle JO et al. Cyclooxygenase 2: a pharmacological target for the prevention of cancer. Lancet Oncol. 2(9), 544-551 (2001).

2. McGettigan P, Henry D. Cardiovascular risk and inhibition of cyclooxygenase: a systematic review of the observational studies of selective and nonselective inhibitors of cyclooxygenase 2. JAMA 296(13), 1633-1644 (2006).

3. Kogiso M, Shinohara T, Dorey CK, Shibata Y. Role of PPAR $\gamma$ in COX-2 activation in mycobacterial pulmonary inflammation. Inflammation 35(5), 1685-1695 (2012).

4. Renna NF, Diez ER, Lembo C, Miatello RM. Role of Cox-2 in vascular inflammation: an experimental model of metabolic syndrome. Mediators Inflamm. 2013, 513251 (2013).

5. Lin C-C, Lee I-T, Yang Y-L, Lee C-W, Kou YR, Yang C-M. Induction of COX-2/PGE(2)/IL-6 is crucial for cigarette smoke extract-induced airway inflammation: role of TLR4-dependent NADPH oxidase activation. Free Radic. Biol. Med. 48(2), 240-254 (2010).

6. Consalvi S, Biava M, Poce G. COX inhibitors: a patent review (2011 - 2014). Expert Opin. Ther. Pat. 25(12), 1357-1371 (2015).

7. Biava M, Porretta GC, Poce G et al. Enlarging the NSAIDs family: ether, ester and acid derivatives of the 1,5-diarylpyrrole scaffold as novel anti-inflammatory and analgesic agents. Curr. Med. Chem. 18(10), 1540-1554 (2011).

8. Patrono C, Patrignani P, García Rodríguez LA. Cyclooxygenase-selective inhibition of prostanoid formation: transducing biochemical selectivity into clinical read-outs. J. Clin. Invest. 108(1), 7-13 (2001).

9. McGettigan P, Henry D. Cardiovascular risk and inhibition of cyclooxygenase: a systematic review of the observational studies of selective and nonselective inhibitors of cyclooxygenase 2. JAMA, 296(13), 1633-44 (2006)

10. Borne R, Mark L, Wilson N. Nonsteroidal anti-inflammatory drugs. In: Foye's Principles of Medicinal Chemistry. Thomas LL, David AW, Victoria FR, Zito W (Eds). Wolters Kluwer, MD, USA, 1021 (2013).

11. García Rodríguez LA, Tacconelli S, Patrignani P. Role of dose potency in the prediction of risk of myocardial infarction associated with nonsteroidal anti-inflammatory drugs in the general population. J. Am. Coll. Cardiol. 52(20), 1628-1636 (2008).

12. Patrignani P, Capone ML, Tacconelli S. NSAIDs and cardiovascular disease. Heart Br. Card. Soc. 94(4), 395-397 (2008).

13. Cingolani G, Panella A, Perrone MG et al. Structural basis for selective inhibition of Cyclooxygenase-1 (COX-1) by diarylisoxazoles mofezolac and 3-(5-chlorofuran-2-yl)-5-methyl-4-phenylisoxazole (P6). Eur. J. Med. Chem. 138, 661-668 (2017). 
14. Carullo G, Galligano F, Aiello F. Structure-activity relationships for the synthesis of selective cyclooxygenase 2 inhibitors: an overview (2009-2016). MedChem Comm8(3), 492-500 (2017).

15. Biava M, Battilocchio $\mathrm{C}$, Poce $\mathrm{G}$ et al. Enhancing the pharmacodynamic profile of a class of selective COX-2 inhibiting nitric oxide donors. Bioorg. Med. Chem. 22(2), 772-786 (2014).

16. Liaras K, Fesatidou M, Geronikaki A. Thiazoles and thiazolidinones as COX/LOX inhibitors. Molecules 23(3), 685 (2018).

17. Bertinaria M, Shaikh MAAG, Buccellati $\mathrm{C}$ et al. Designing multitarget anti-inflammatory agents: chemical modulation of the lumiracoxib structure toward dual thromboxane antagonists-COX-2 inhibitors. ChemMedChem 7(9), 1647-1660 (2012).

18. Chennamaneni S, Zhong B, Lama R, Su B. COX inhibitors indomethacin and sulindac derivatives as antiproliferative agents: synthesis, biological evaluation, and mechanism investigation. Eur. J. Med. Chem. 56, 17-29 (2012).

19. ClinicalTrials website. https://clinicaltrials.gov/

20. Todoric J, Antonucci L, Karin M. Targeting inflammation in cancer prevention and therapy. Cancer Prev Res. 9(12), 895-905 (2016). 